Check for updates

Cite this: RSC Adv., 2017, 7, 35096

\title{
The heterogeneous selective reduction of PHB as a useful method for preparation of oligodiols and surface modification
}

\author{
Paweł Chaber, Michał Kwiecień, (D) Magdalena Zięba, Michał Sobota \\ and Grazyna Adamus (DD*
}

A selective heterogeneous reduction of natural PHB with lithium borohydride as a reducing agent has been described. Despite the method proceeding in a heterogeneous way, it allows obtaining hydroxyl terminated oligomers with low polydispersity, controlled average molecular weight, and defined end groups. The structure of resulting oligomers has been proven by ${ }^{1} \mathrm{H}$ NMR and mass spectrometry. This method would constitute a source of PHB oligodiols useful in the synthesis of new tailor-made biomaterials. It has also been demonstrated that this simple method may be used to modify the surface chemistry of the polyester by generating free hydroxyl groups on the outermost face. The presence of hydroxyl groups on the polymer surface was confirmed by ATR-FTIR studies as well as contact angle measurement. As expected, the change in surface chemistry improved the hydrophilicity of the polymer surface. Since surface properties are critically important for cell-material interactions, this kind of modification may be a very useful tool in tissue engineering.

Received 1st June 2017

Accepted 5th July 2017

DOI: 10.1039/c7ra06111k

rsc.li/rsc-advances

The most common and studied among PHAs polymer, poly(3-hydroxybutyrate) (PHB), is very stiff and brittle and has low elasticity. ${ }^{9}$ The stiffness of PHB is a result of its high crystallinity (about 80\% (ref. 10)), which, in turn, arises from its regular structure. PHB is, namely, a stereoregular homopolyester with an $\mathrm{R}$ configuration at all the $\beta$-carbons, so the polymer is completely isotactic. Moreover, its processing is difficult as it readily undergoes thermal degradation at temperatures close to the melting point. ${ }^{11}$ Fortunately, all these difficulties may be overcome since the mechanical and thermal properties of PHAs are greatly affected by the monomer structure and copolymer composition.,52,13 Therefore, by changing the chemical structure of the alkyl side chain or by incorporating other hydroxyalkanoic acid co-monomer units into the main chain of PHAs, it is possible to create new polymers with desired properties.

In general, biotechnological or chemical approach can be applied to modify the chemical composition of the bacterial polyesters. Since they are synthesised in nature by bacterial fermentation, the variation in the chemical structure can be achieved by using appropriate microorganism type and growth superior biodegradability and biocompatibility., ${ }^{4,5}$ Owing to these properties, PHAs could be employed as biomaterials for tissue engineering and controlled drug delivery., ${ }^{\mathbf{4}, \mathbf{8}}$

PHAs certainly possess many interesting properties, but they are not without disadvantages. Their major shortcoming are their low mechanical properties. The difficulty of PHAs processing is another drawback which hinders their practical use.

Fig. 1 General chemical structure of PHAs. In the figure, $n=1-4, x=$ 200-12 000, and R is a hydrogen atom or an alkyl group. 
conditions. ${ }^{\mathbf{1} 8}$ However, the bacterial enzymes simply accept only certain hydroxyalkanoic acids in the course of polymerisation. Thus, the biotechnological process leads to materials with structures similar only to those shown in Fig. 1, and hence to materials with limited thermomechanical properties. As a result, the microbial polyesters often do not meet the very specific requirements set to biomaterials in different medical applications.

Systematic research has been conducted on the development of new polymeric biomaterials based on structural fragments derived from microbial PHAs. Those structural fragments are very interesting building blocks for the synthesis of more sophisticated polymer architectures with tailored properties. They can be obtained using i.e. chemical reactions such as controlled degradation processes. In the case of structural segments, the end groups, which are usually ignored for longchain PHAs, gain a significant importance since they determine the possibility of their further use in the synthesis of new materials.

Several attempts have been made to obtain structural fragments from PHAs. ${ }^{\mathbf{1 4 - 2 6}}$ A molecular mass reduction of PHA may be achieved by various methods, such as an acid-catalysed alcoholysis (a transesterification reaction), ${ }^{\mathbf{1 4 - 1 8}}$ an acid or a base hydrolysis, ${ }^{19}$ a saponification reaction, ${ }^{20,21}$ a thermal degradation, ${ }^{22,23}$ or a reduction reaction. ${ }^{24,25}$ All these methods lead to obtaining low-molecular-weight PHAs with well-defined end groups that can take part in further polymerisation. In the case of a saponification reaction and a thermal degradation, the formation of oligomers with olefinic and carboxylic end groups is observed. ${ }^{20,21}$ Those structural fragments could be polymerised to yield polymers via free-radical methods, but nevertheless, Nguyen et al. reported some difficulties in applying these chain-extending reactions. Much work has been done to obtain diol ended PHAs, particularly hydroxyl terminated PHB, which present some advantages over the other PHAs family members. Oligomers terminated by free hydroxyl groups are very useful in the chemical synthesis as they can react, for example, with diisocyanate monomers to form various polyurethanes, or with long-chain dicarboxylic acids to increase the distance between the ester linkages in the newly formed polymeric chains. For this purpose oligodiols of PHB are characterized with a lower steric hindrance, which results in higher reactivity in the above-mentioned reactions. PHB oligomers with reactive hydroxyl ends can be prepared by two methods: via transesterification reaction, ${ }^{\mathbf{1 4}-18}$ and by borohydride reduction. ${ }^{24,25}$ In the first case 1,4-butanediol, ethylene glycol, and glycerol were employed, and in the second - sodium borohydride $\left(\mathrm{NaBH}_{4}\right)$ and lithium borohydride $\left(\mathrm{LiBH}_{4}\right)$ were used as reducing agents. Montoro compared both methods and concluded that the borohydride reduction was more efficient. ${ }^{26}$ A borohydride reduction of PHB has a significant advantage over a transesterification reaction because the latter is limited in terms of molar masses of the synthesised oligomers. The first could even result in the monomeric units.

Despite the fact that history of hydride reductions began over eighty years ago with a study of the reactions of diborane $\left(\mathrm{B}_{2} \mathrm{H}_{6}\right),{ }^{27}$ borohydride reductions of polyesters are not fully investigated. Bergamaschi et al. reported that the treatment of PHB (dissolved in chloroform) with sodium borohydride leads to the formation of oligomers terminated with hydroxyl groups, but these products were not the only ones present in the postreaction mixture. Low-molecular-weight $\mathrm{PHB}$ with an unsaturated and a carboxylic end group were also observed, with the latter being dominant. ${ }^{24}$ Recently, we have developed a highly selective method for a controlled degradation of some PHAs, via a reduction reaction with lithium borohydride. ${ }^{25}$ However, despite all advantages the method has, it only applies to those PHAs which are soluble in solvents appropriate for lithium borohydride reduction (in tetrahydrofuran or diethyl ether). Since the natural PHB is insoluble in almost all common organic solvents, the reduction of PHB by using the abovementioned method has failed.

Here, we report a novel heterogeneous method of the reduction of sparingly soluble PHAs on the example of the most well-known among them, the PHB. The method allows obtaining low-molecular-weight PHB diols in a satisfactory yield at room temperature. In addition, the obtained oligomers have a high purity proved by ${ }^{1} \mathrm{H}$ NMR and ESI-MS ${ }^{n}$ analysis. Moreover, the elaborated method can be used to modify the chemistry of the surface layer of polyester devices fabricated, for example, by extrusion moulding. What is worth noting is that the modification of the surface proceeds under very mild conditions. It is also shown that elaborated method is useful to increase the hydrophilicity of the modified surface. The surface itself holds great importance in the cell-material interactions and to a great extent determines the biological performance of a biomaterial. It controls such important processes as cell adhesion and growth, processes which largely define success or failure of, for example, producing a scaffold. ${ }^{28,29}$

\section{Experimental section}

\section{Materials}

Poly(3-hydroxybutyrate) (PHB) was provided by the Biomer Company (Germany). In order to remove biological impurities the sample of raw PHB was purified by reprecipitation from its chloroform solution in hexane. Next, the obtained powder was dried in vacuum at room temperature and then it was ground in a mortar to average powder granulation. The sample of PHB was characterized by gel permeation chromatography (GPC) and ${ }^{1} \mathrm{H}$ and ${ }^{13} \mathrm{C}$ NMR. The number-average molar mass of $\mathrm{PHB}$, determined by GPC, was $228000 \mathrm{~g} \mathrm{~mol}^{-1}$ with a polydispersity of 17.7.

Lithium borohydride, $\mathrm{LiBH}_{4}$, was purchased as a solution (as a 2.0 $\mathrm{M}$ solution in THF and a $0.5 \mathrm{M}$ solution in diethyl ether) from Aldrich as well as phosphoric acid, $\mathrm{H}_{3} \mathrm{PO}_{4}$, (85 wt\% in $\left.\mathrm{H}_{2} \mathrm{O}, \mathrm{FG}\right)$. Hydrochloric acid ( $\mathrm{HCl}$ ) solution in $\mathrm{H}_{2} \mathrm{O}(35-38 \%$, pure p. a., Avantor), the hydrochloric acid solution in diethyl ether (2.0 M, Aldrich) and chloroform, $\mathrm{CHCl}_{3},(98.5 \%$, pure p. a., Avantor) were used as received. Tetrahydrofuran, THF, (pure, Avantor), diethyl ether, DE, (pure, Avantor), toluene (pure, Avantor) were purified prior to use. THF and diethyl ether were distilled over potassium-sodium alloy and stored in Schlenk flasks under an argon atmosphere. Toluene was distilled from calcium hydride and stored over $4 \mathrm{~A}$ molecular sieves. 


\section{Methods}

Preparation of oligodiols. PHB oligodiols were obtained via the reduction of the ester linkages of PHB biopolyester at room temperature. The starting polymer was immersed in the THFtoluene solvent mixture and then lithium borohydride $\left(\mathrm{LiBH}_{4}\right)$ was added.

Borohydride reduction of PHB powder. To a round-bottom flask, which was evacuated and refilled with argon, the natural PHB in powder form $(0.2500 \mathrm{~g})$ was added along with a magnetic stirrer, THF $(12.0 \mathrm{~mL})$ and toluene $(12.5 \mathrm{~mL})$. The flask was equipped with a septum, and the heterogeneous mixture was then stirred at room temperature for $0.5 \mathrm{~h}$. After this time a $2.0 \mathrm{M}$ solution of lithium borohydride in THF $(0.4 \mathrm{~mL}, 8 \mathrm{mmol})$ was introduced with a syringe to the flask and the reaction mixture was allowed to stir for $4 \mathrm{~h}$. The reduction was interrupted by adding an aqueous solution of $10 \%$ phosphoric acid (always $0.8 \mathrm{~mL}$ ) and stirring for another $0.5 \mathrm{~h}$, after which the solvents were evaporated under vacuum. In order to isolate the obtained oligodiols, chloroform $(20 \mathrm{~mL})$ was subsequently added to the white residue, and the resulting hazy mixture was stirred for at least $0.5 \mathrm{~h}$. The insoluble inorganic compounds (including lithium phosphate and lithium hydrogen phosphates) were then removed by simple filtration. Additionally, the filter cake was washed with another portion of chloroform $(5 \mathrm{~mL})$. The clear chloroform solution was then transferred into a separator funnel and washed one time with an aqueous solution of $0.5 \mathrm{M} \mathrm{HCl}(25 \mathrm{~mL})$ and five times with an aqueous solution of $1 \mathrm{M} \mathrm{NaCl}(25 \mathrm{~mL})$. Finally, the organic layer was evaporated to dryness giving $0.1855 \mathrm{~g}(74.2 \%)$ of PHB oligodiols.

Preparation of PHB discs. The discs from natural PHB were prepared by injection molding method (Thermo Scientific HAAKE MiniLab II, an extruder machine and HAAKE MiniJet, a mini injection molding machine). PHB was warmed up close to the melting point $\left(170{ }^{\circ} \mathrm{C}\right)$ and then was formed in diskshaped samples.

Borohydride reduction of PHB discs' surface. Ten discs made of natural PHB were transferred to a round bottom flask equipped with a magnetic stir bar. Then the flask was evacuated and refilled with argon. $6.4 \mathrm{~mL}$ of dry diethyl ether was then added in an argon flow. The gas inlet adapter was replaced by a septum and $3.6 \mathrm{~mL}$ of a $0.5 \mathrm{M}$ solution of lithium borohydride in diethyl ether was added through the septum via syringe. The reaction mixture consists of discs immersed in diethyl ether was stirred at room temperature for 24 hours, after which the solvent was decanted. The discs were washed two times with $5 \mathrm{~mL}$ of dry diethyl ether. Thereafter, $5 \mathrm{~mL}$ of the solution of $\mathrm{HCl}$ in diethyl ether was introduced to the flask. The discs were being washed with $\mathrm{HCl}$ solution for another 30 minutes. The ethereal solution of $\mathrm{HCl}$ was decanted and the discs were washed three times with $5 \mathrm{~mL}$ of dry diethyl ether. Finally, PHB round-shaped devices were dried for 72 hours in a desiccator in vacuo with $\mathrm{P}_{2} \mathrm{O}_{5}$ on the bottom. Six of the discs were firstly measured by ATR-FTIR spectroscopy and then the water contact angles were measured as well.

In parallel to the functionalization of PHB discs' surfaces, a blind test was performed. The protocol of the test was the same as above except that herein lithium borohydride was not added. The discs were only just immersed in the solvent. After 24 hours they were treated with hydrochloric acid, washed with diethyl ether and finally dried over 72 hours.

GPC analysis. The number-average molar mass $\left(M_{\mathrm{n}}\right)$ and the molecular mass distribution index $\left(M_{\mathrm{w}} / M_{\mathrm{n}}\right)$ of the plain PHA samples were determined by GPC that was conducted in a $\mathrm{CHCl}_{3}$ solution at $35{ }^{\circ} \mathrm{C}$ with a flow rate of $1 \mathrm{~mL} \mathrm{~min}^{-1} \mathrm{using}$ a Spectra-Physic 8800 solvent delivery system. This system had a set of two PLgel $5 \mu \mathrm{m}$ MIXED-C ultra-high efficiency columns $\left[M_{\mathrm{w}}\right.$ range $-200-2000000 \mathrm{~g} \mathrm{~mol}^{-1}$; efficiency (1/2 ht) (plates per m) 56 593] and a Shodex SE 61 refractive index detector. For the GPC analysis of the PHA oligodiols, a PLgel $3 \mu \mathrm{m}$ Mixed-E column $\left[M_{\mathrm{w}}\right.$ range up to $25000 \mathrm{~g} \mathrm{~mol}^{-1}$; efficiency (1/2 ht) (plates per m) 98 869] and THF, as the solvent, were used. A sample solution volume of $10 \mu \mathrm{L}$ (concentration of $1 \% \mathrm{w} / \mathrm{v}$ ) was injected. Polystyrene standards with a narrow molecular mass distribution were used to generate calibration curves.

NMR analysis. The ${ }^{1} \mathrm{H}$ NMR spectra were recorded using a Bruker-Avance II $600 \mathrm{MHz}$ with Ultrashield Plus Magnets. The ${ }^{1} \mathrm{H}$ spectra were run with $\mathrm{CDCl}_{3}$ as the solvent and using tetramethylsilane (TMS) as an internal standard. The ${ }^{13} \mathrm{C}$ NMR spectra were recorded on a Bruker-Avance II $150 \mathrm{MHz}$, using $\mathrm{CDCl}_{3}$ and TMS. ${ }^{1} \mathrm{H}$ NMR spectra were obtained with 64 scans, an 11 ls pulse width and $2.65 \mathrm{~s}$ acquisition time, whereas ${ }^{13} \mathrm{C}$ NMR spectra were obtained with 20480 scans, a 9.40 ls pulse width, and $0.9088 \mathrm{~s}$ acquisition time.

\section{ESI-MS $^{n}$ experiments}

Electrospray mass spectrometry (ESI-MS ${ }^{n}$ ) analysis. Electrospray mass spectrometry analysis was performed using a Finnigan LCQ ion trap mass spectrometer (Finnigan, San Jose, CA, USA). The polyester samples were dissolved in a chloroform/ methanol system $(1: 1 \mathrm{v} / \mathrm{v})$, and the solutions were introduced into the ESI source by continuous infusion using the instrument's syringe pump at a rate of $10 \mu \mathrm{L} \min ^{-1}$. The LCQ ESI source was operated at $4.5 \mathrm{kV}$, and the capillary heater was set to $200{ }^{\circ} \mathrm{C}$. Nitrogen was used as the nebulising gas. For ESI-MS/MS experiments, the ions of interest were isolated monoisotopically in the ion trap and were collisionally activated. The helium damping gas that was present in the mass analyser acted as a collision gas. The $\mathrm{RF}$ amplitude, which had a significant voltage range, was set to a value that caused the peak height of the molecular ion to decrease by at least $50 \%$. The analysis was performed in the positive-ion mode.

ATR-FTIR analysis. Fourier transform infrared (FTIR) spectra were recorded on Jasco FT-IR-6700 (Jasco Corporation, Tokyo, Japan) spectrometer equipped with ATR accessory. 64 scans were accumulated at $2 \mathrm{~cm}^{-1}$ resolution in the region between 4000 and $400 \mathrm{~cm}^{-1}$.

\section{Contact angle measurements}

The goniometer. Contact angle measurements $(\Theta)$ for PHB discs' surface were measured statically using a contact angle goniometer (CAM 101 from KSV instruments) equipped with a temperature control unit. The water contact angles were determined in air using the sessile drop method. A water drop 
of volume around $5 \mu \mathrm{L}$ was suspended from the syringe needle and brought into contact with the surface of the discs. A series of images were acquired during $30 \mathrm{~s}$. Contact angles were measured after the drop was stabilized. The contact angle measurements were performed for a dry surface. The average contact angle values and standard deviations were obtained by measuring the contact angle at one position of each of six discs.

\section{Results and discussion}

Application of lithium borohydride as efficient reducing agent for oligodiols preparation from selected PHA polyester was reported recently. ${ }^{25}$ However, most commonly members of PHA family are highly crystalline and their solubility in solvents suitable for the reduction process is limited. Therefore, we have tried to conduct the reduction of $\mathrm{PHB}$ in two phase system. $\mathrm{PHB}$ is the easiest available and cheapest member of PHA family but has also several disadvantages which limit its wide using, so the possibility of its modification seems to be a good objective intentional. The general scheme of proposed modification is presented on Scheme 1.

In our previous work concerning reduction process of soluble PHA biopolyesters, THF was used as a suitable solvent. At the beginning of this work, the possibility of the use of other solvents for two-phase reduction process was investigated. Four different solvents or mixtures of solvents: tetrahydrofuran (THF) diethyl ether (DE), THF : toluene $(1: 1)$ and DE : toluene $(1: 1)$ were considered and the results of this investigation are presented in Table 1 . The results indicated that the solvent which allows obtaining oligomers with lowest average molar mass in the same conditions (reaction were carried out for $4 \mathrm{~h}$, using $0.8 \mathrm{mmol} \mathrm{LiBH}_{4}$ in $25 \mathrm{~mL}$ of solvent(s) was a mixture of THF and toluene in ratio $(1: 1))$. Therefore, in next steps of our research concerning two-phase reduction process of PHB using lithium borohydride as reducing agent, this mixture was used as a solvent.

In the next step, the time necessary to conduct an efficient reaction of obtaining oligomers with low average molar mass was determined. The results of this study are presented in Table 2 . The reaction was carried out in the range of 0.5 to 6 hours. The highest decrease of average molar mass of starting biopolyester is observed after $0.5 \mathrm{~h}$ what testifies that this process is relatively fast. During elongation of time of reaction, slow decrease of average molar mass is observed up to $4 \mathrm{~h}$. Further time elongation (over $4 \mathrm{~h}$ ) does not result in further decrease in average molar mass, therefore the $4 \mathrm{~h}$ were determined as sufficient time for this reduction process.

Finally, the influence of the amount of reducing agent $\left(\mathrm{LiBH}_{4}\right)$ on the decrease of average molar mass of starting PHB
Table 1 PHB oligomers obtained by borohydride reduction of high molar mass PHB biopolyester ${ }^{a}$

\begin{tabular}{lllll}
\hline No. & Solvent & $M_{\mathrm{n}}\left[\mathrm{g} \mathrm{mol}^{-1}\right]$ & $M_{\mathrm{w}} / M_{\mathrm{n}}$ & Yield [\%] \\
\hline 1 & THF & 4000 & 1.71 & 80.2 \\
2 & DE & 4000 & 1.70 & 83.5 \\
3 & THF : toluene $(1: 1)$ & 2800 & 1.49 & 74.2 \\
4 & DE : toluene $(1: 1)$ & 3800 & 1.70 & 83.4
\end{tabular}

${ }^{a}$ The reactions were carried out for 4 hours with $8 \mathrm{mmol}$ of $\mathrm{LiBH}_{4}$ in 25 $\mathrm{mL}$ of solvent(s). The number average molar mass of PHB was $228000 \mathrm{~g}$ $\mathrm{mol}^{-1}$ with polydispersity of 17.7 .

biopolyester and the yield of synthesized PHB oligodiols were also investigated. The results are summarized in Table 3. Obtained results show that increasing the amount of lithium borohydride above the $0.8 \mathrm{mmol}$ is purposeless because it does not lead to a further decrease of PHB oligodiols average molar mass.

Simultaneously, a decrease of yield of reaction with decreasing of average molar mass is observed. The observed decrease of yield of reaction may be caused by PHB oligodiols purification process after the reaction. The very low molar mass PHB oligodiols may be washed out by water which is used during purification. Additionally, in the case of two-phase reduction at the end of the process we have two fractions of $\mathrm{PHB}$ in the reaction mixture, the first one is not soluble and the second one (PHB oligomers with very low molar mass) is soluble in the solvent. This situation leads to competition between reactions proceeding in solution and on phase boundary. Reaction in solution proceeded faster and produced PHB oligomers with very low average molar mass which were soluble in water and could be removed during the purification. The increase of reducing agent dose did not influence on the further decrease average molar masses of separated PHB oligodiols.

For the structural characterization of the obtained oligodiols, ${ }^{1} \mathrm{H}$ NMR and mass spectrometry were used.

Table 2 PHB oligomers obtained after different time of the reduction reaction of $\mathrm{PHB}$ biopolyester ${ }^{a}$

\begin{tabular}{lllll}
\hline No. & $t[\mathrm{~h}]$ & $M_{\mathrm{n}}\left[\mathrm{g} \mathrm{mol}^{-1}\right]$ & $M_{\mathrm{w}} / M_{\mathrm{n}}$ & Yield [\%] \\
\hline 1 & 0.5 & 4700 & 1.75 & 86.6 \\
2 & 1 & 3500 & 1.59 & 80.7 \\
3 & 2 & 3200 & 1.60 & 77.1 \\
4 & 4 & 2800 & 1.49 & 74.2 \\
5 & 6 & 2800 & 1.49 & 72.0
\end{tabular}

${ }^{a}$ The reactions were carried out with $8 \mathrm{mmol}$ of $\mathrm{LiBH}_{4}$ in $25 \mathrm{~mL}$ of the THF-toluene solvent mixture. The number-average molar mass of PHB was $228000 \mathrm{~g} \mathrm{~mol}^{-1}$ with polydispersity of 17.7 .

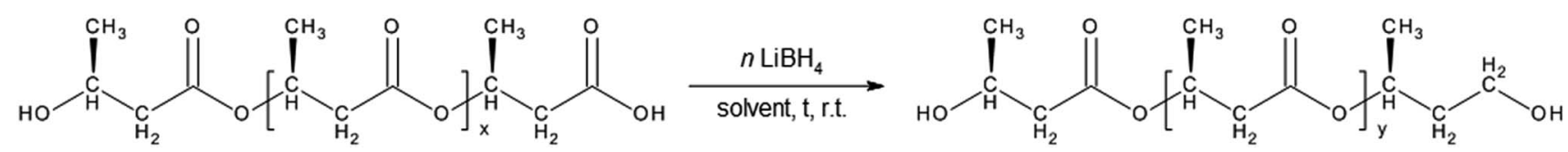

Scheme 1 The reduction reaction of PHB biopolyester with lithium borohydride. In the scheme, $t$ means time and $y \ll x$. 
Table 3 PHB oligomers obtained by using different amount of the reducing agent ${ }^{a}$

\begin{tabular}{lllll}
\hline No. & $\begin{array}{l}\text { Amount of } \mathrm{LiBH}_{4} \\
{[\mathrm{mmol}]}\end{array}$ & $M_{\mathrm{n}}\left[\mathrm{g} \mathrm{mol}^{-1}\right]$ & $M_{\mathrm{w}} / M_{\mathrm{n}}$ & $\begin{array}{l}\text { Yield } \\
{[\%]}\end{array}$ \\
\hline 1 & 0.2 & 3900 & 1.70 & 76.6 \\
2 & 0.4 & 3400 & 1.62 & 76.3 \\
3 & 0.6 & 3400 & 1.68 & 81.8 \\
4 & 0.8 & 2800 & 1.49 & 74.2 \\
5 & 1.0 & 2900 & 1.51 & 73.7 \\
6 & 1.2 & 2900 & 1.52 & 73.3
\end{tabular}

${ }^{a}$ The reactions were carried out in $25 \mathrm{~mL}$ of the THF-toluene solvent mixture, for 4 hours. The number-average molar mass of PHB was $228000 \mathrm{~g} \mathrm{~mol}^{-1}$ with polydispersity of 17.7 .

\section{Proton nuclear magnetic resonance ( ${ }^{1} \mathrm{H}$ NMR) spectroscopy}

The ${ }^{1} \mathrm{H}$ NMR spectrums of the oligomers obtained via the selective reduction of PHB biopolyester are presented in Fig. 2. The main signals (marked as $1,2,3$ ) in the ${ }^{1} \mathrm{H}$ NMR spectrum correspond to the protons of the 3-hydroxybutyrate repeating units. Additionally, in the presented spectrum signals corresponding to the protons of the end groups of obtained oligomers are observed. Thus, the signals denoted a, b, and c were assigned to the protons of the 3-hydroxybutyrate end group, whereas the signals denoted $\mathrm{d}, \mathrm{e}, \mathrm{f}$, and $\mathrm{g}$ were assigned to the protons of the 1-methyl-3-hydroxypropyl end group. In the ${ }^{1} \mathrm{H}$ NMR spectrum any additional signals besides those from oligodiols are not observed, thus this analysis confirmed selectivity of the reduction reaction of PHB biopolyester (Fig. 2).

\section{Electrospray ionization mass spectrometry (ESI-MS)}

ESI-MS is a very useful technique for detailed analysis of synthetic polymers. This sensitive and non-averaging technique provides detailed information about individual oligomer chains as well as the chemical structure of their end groups. ESI-MS is used successfully for structural characterization PHA biopolyesters and other polyesters and copolyesters. ${ }^{21,25,30-32}$ Therefore, this technique was also used for the structural characterization of the obtained PHB oligodiols.

Fig. 3 shows the ESI-MS spectrum of PHB oligomers obtained via selective reduction of high molar mass PHB biopolyester. The ions in the main series in ESI-MS spectrum occurred regularly at $86 \mathrm{Da}$ what corresponds to the 3-hydroxybutyrate constitutional unit. Thus, the main series of ions present in the mass spectrum (Fig. 3) (marked as A) can be assigned to the sodium adduct of PHB oligomers. For further structural assignment of PHB oligomers, tandem mass spectrometry (ESI-MS/MS) was used. To verify the structure of individual PHB oligomers chains, ESI-MS/MS fragmentation experiments were performed for selected ions isolated from Series A.

The ESI-MS/MS spectrum shown in Fig. 4, was obtained for the precursor ions at $m / z 1231$, which was selected from ESI-MS spectrum (Fig. 3) of PHB oligomers obtained via selective reduction of $\mathrm{PHB}$ biopolyester.

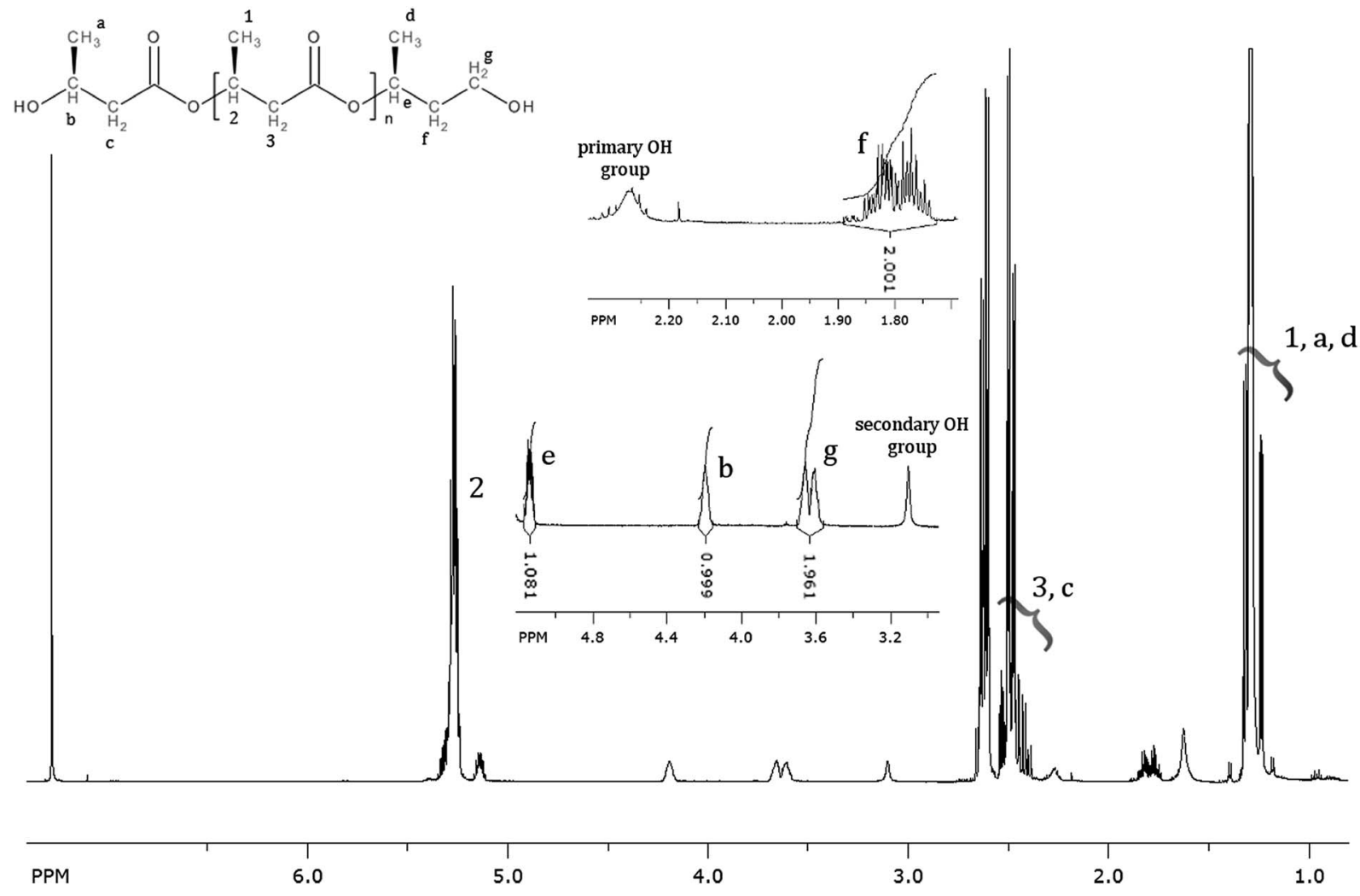

Fig. $2{ }^{1} \mathrm{H}$-NMR spectrum of PHB oligomers obtained through reduction with lithium borohydride. 


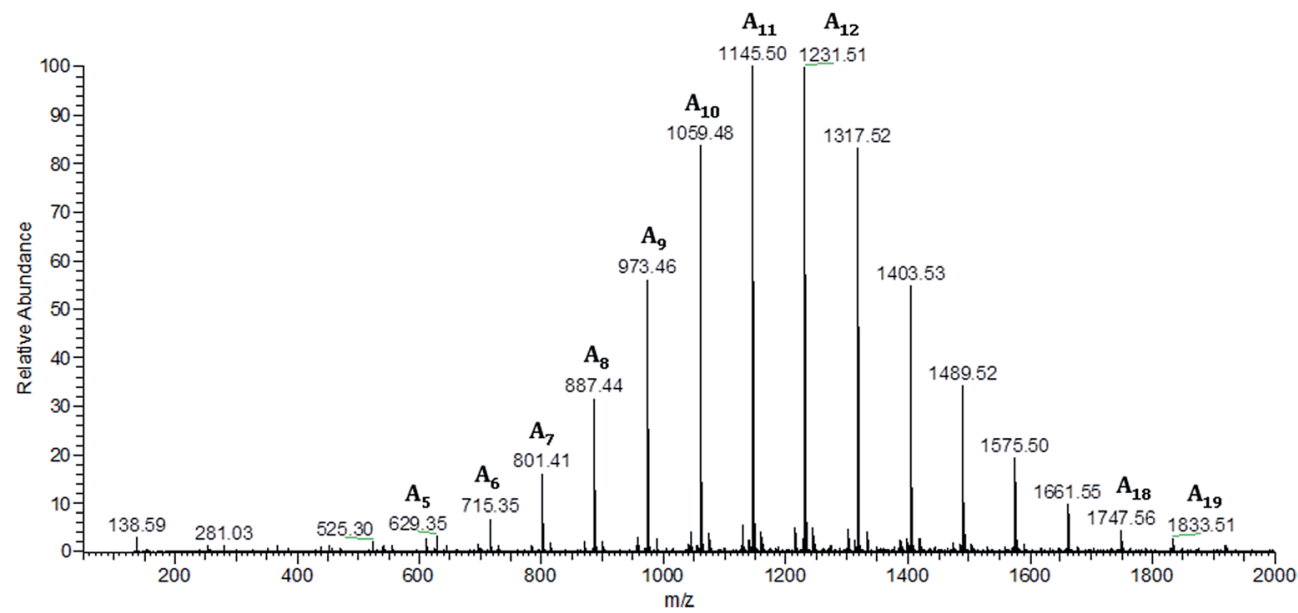

Fig. 3 ESI-MS spectrum (positive ion mode) of the oligodiols obtained via the selective reduction of a PHB biopolyester.

The fragmentation of this ion, which occurs as a result of the statistical breakage of the ester bonds along PHB oligodiols chain, leads to the formation of two series of product ions. The series of product ions, at $\mathrm{m} / \mathrm{z} 1127,1041,955,869,783,697$ and 611, terminated by crotonic and carboxylic end group are created: first by the expulsion of 3-hydroxybutanoic acid (104 Da) and further by the loss of crotonic acid molecules. The second series of product ions at $\mathrm{m} / z 1159,1073,987,901,815$, 729 and 643 terminated by 3-hydroxybutyrate and carboxylic end group is formed in the first step by the loss of 2-butenyl alcohol (crotonyl alcohol; $72 \mathrm{Da}$ ) and in next steps by expulsion of crotonic acid molecules (86 Da).
Thus, the ESI-MS ${ }^{2}$ experiments performed for selected sodium adducts of PHB oligomers confirmed that the obtained PHB oligomers contained two hydroxyl end groups.

Application of NMR and ESI-MS technique for determination of the chemical structure of the obtained oligomers allows confirming that selective reduction of polyester may be carried out also in the heterogeneous system. Limited solubility of polyester in a solvent did not influence the selectivity of the reduction process using lithium borohydride as reducing agent. Moreover, in some range, the average molar mass of the resulting PHB oligodiols may be controlled by the amount of reducing agent used in the reaction. Very interesting results of

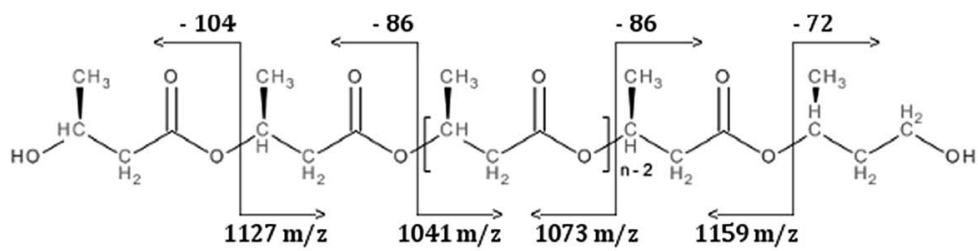

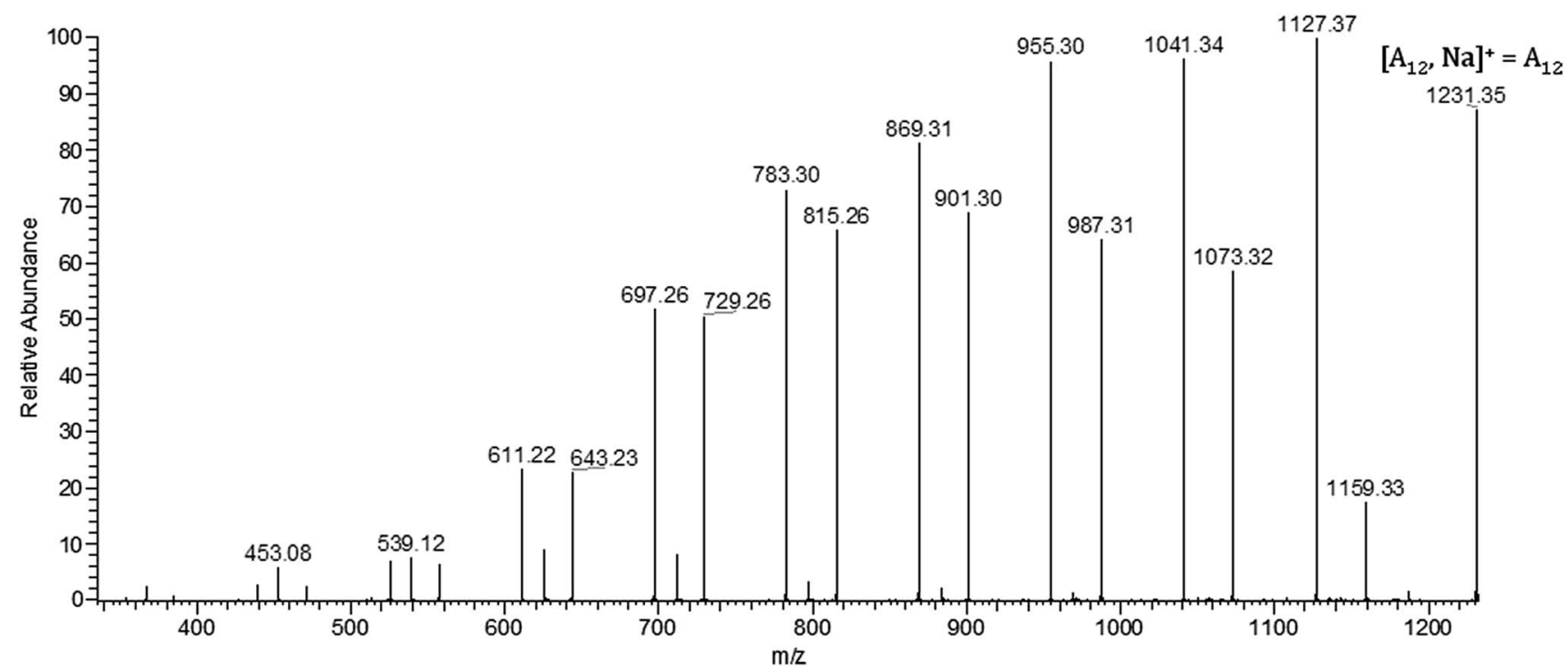

Fig. 4 ESI-MS/MS product ion spectra and fragmentation pathways of the sodium adduct at $\mathrm{m} / \mathrm{z} 1231$. 

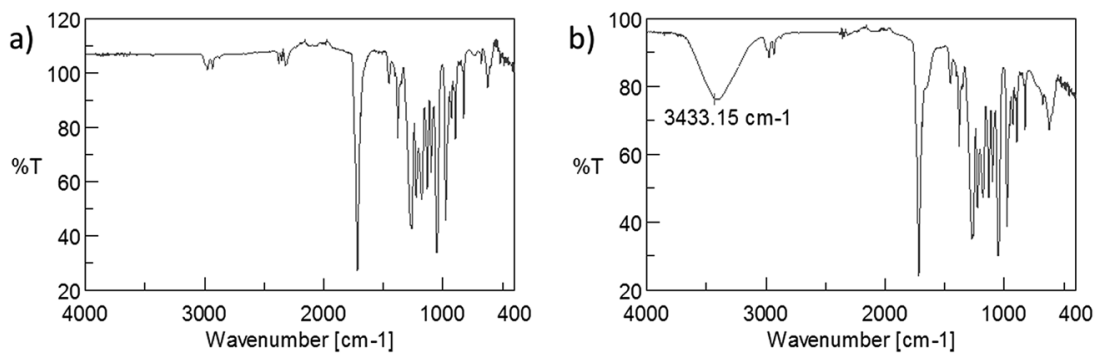

Fig. 5 ATR-FTIR spectra of (a) PHB surface of blind sample and (b) chemically modified PHB surface.

a)

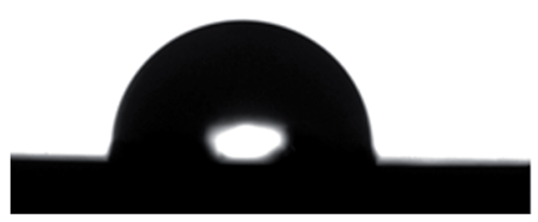

b)

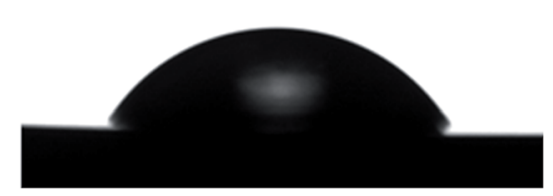

Fig. 6 Contact angle images of water on (a) $\mathrm{PHB}$ surface untreated with lithium borohydride (the blind sample; $\left.\theta=90.6^{\circ}\right)$ and $(\mathrm{b}) \mathrm{LiBH}_{4}$-treated PHB surface $\left(\theta=58.9^{\circ}\right)$.

studies about the heterogeneous reduction of PHB biopolyester have inspired us to apply this method for the modification of the surface chemistry of polyester materials. Surfaces bearing polar functional groups offer enhanced biological performance by improving cell attachment to the surface ${ }^{28,33}$ as well as cell growth. ${ }^{34}$ Regarding the reduction of PHB powder, which leads to obtaining oligodiols, the functionalization of the surface of polymer devices with much smaller surface area could generate hydroxyl groups on the outermost face without causing any visible destruction in the modified material. In fact, using lithium borohydride as the reducing agent generates free hydroxyl groups on the outermost face of PHB devices. Discs fabricated by extrusion of PHB were used as a model of the surface. As it is shown in Table 2, the reduction reaction of the powdered PHB proceeds in THF as well as in diethyl ether. Regarding the discs, our goal was not to obtain the oligomers with the lowest mass possible, but just to functionalize the surface. Thus, we decided to perform the borohydride reduction of PHB devices in diethyl ether instead of in THF-toluene system due to at least two facts. Firstly, diethyl ether is highly volatile, and therefore it is easier to evaporate from the surface. Secondly, diethyl ether is employed as a surface antiseptic and cleansing agent, ${ }^{35}$ so PHB devices after the functionalization could be instantly used in medical applications. To confirm that the functionalization of the surface proceeds efficiently, ATRFTIR analyses were performed. The ATR-FTIR spectra are presented in Fig. 5.
ATR-FTIR spectrum for extruded discs before reduction process looks like typically FTIR spectrum for PHB biopolyester. In the spectrum for disc after reduction process (presented in Fig. $4 \mathrm{~b}$ ) wide bands in the range of $3000-3700 \mathrm{~cm}$ are observed. This simple and fast analysis confirms presence of the hydroxyl group on the surface of discs. Generation of free hydroxyl groups on the surface of extruded discs from PHB is possible, however the longer time of reaction than in the case of powder reduction is required. The presence of free hydroxyl groups on the surface causes an increase of hydrophilicity what is very important in cell growing process. For determination of increase of hydrophilicity of the surface after the reduction process, the measurement of contact angle was performed. The pictures of a drop on the surface before and after the reduction reaction are presented in Fig. 6 .

The surface untreated with the reducing agent showed noticeably higher contact angle value compared to the chemically modified surface with the average values of $90.3^{\circ} \pm 4.8^{\circ}$ and $59.5^{\circ} \pm 1.4$ respectively. The results show the increase in surface hydrophilicity. Strictly speaking, the hydrophobic PHB surface becomes hydrophilic.

\section{Conclusions}

A heterogeneous system which consists of solid PHB and a solution of lithium borohydride appears to be an efficient method for preparation of PHB oligodiols. Despite the fact that this reaction is a phase boundary reaction, it is quite selective 
and leads to obtaining oligomers with low polydispersity and defined end groups. The average molar masses of the obtained hydroxyl terminated oligomers can be controlled to some extent by changing the amount of reducing agent. Moreover, the results of the present investigation revealed that the developed method is useful to modify surface chemistry. Discs extruded from natural PHB and immersed to the lithium borohydride solution bear hydroxyl groups on the surface. Those hydroxyl groups appeared in the IR spectrum. Furthermore, the contact angle measurements showed that the surface becomes more hydrophilic as a result of the reduction process.

The developed by us method of heterogeneous selective reduction of sparingly soluble PHAs constitute the extension of selective reduction of PHA biopolyesters soluble in standard solvents used for reduction process, recently reported by us. ${ }^{25}$ The elaborated method allows to obtain uniform PHB diols from PHB biopolyester that is produced on large scale and easily available on the market. The obtained low-molecular-weight PHB diols are useful for the further synthesis of new biodegradable polymeric materials with tailored properties.

In addition, the elaborated method can be used to modify the hydrophilicity of the surface layer of polyester devices fabricated from PHB.

Thus, this easy method seems to be useful for application in tissue engineering for creation free hydroxyl group on the surface, e.g. three-dimensional scaffold for cell growth.

Preliminary biological studies proving the usefulness of the elaborated method in the preparation of scaffolds will be the subject of our further work.

\section{Acknowledgements}

This work was supported by the National Science Centre Poland: contract No. UMO-2013/11/B/ST5/02222.

\section{References}

1 A. Anjum, M. Zuber, K. M. Zia, A. Noreen, M. N. Anjum and S. Tabasum, Microbial production of polyhydroxyalkanoates (PHAs) and its copolymers: A review of recent advancements, Int. J. Biol. Macromol., 2016, 89, 161-174.

2 A. Steinbüchel and H. E. Valentin, Diversity of bacterial polyhydroxyalkanoic acids, FEMS Microbiol. Lett., 1995, 128(3), 219-228.

3 A. K. Singh and N. Mallick, SCL-LCL-PHA copolymer production by a local isolate, Pseudomonas aeruginosa MTCC 7925, Biotechnol. J., 2009, 4(5), 703-711.

$4 \mathrm{~S}$. F. Williams and D. P. Martin, Applications of Polyhydroxyalkanoates (PHA) in Medicine and Pharmacy, in Biopolymers Online, Wiley-VCH Verlag GmbH \& Co. KGaA, 2005.

5 S. Khanna and A. K. Srivastava, Recent advances in microbial polyhydroxyalkanoates, Process Biochem., 2005, 40(2), 607619.

6 M. Zinn, B. Witholt and T. Egli, Occurrence, synthesis and medical application of bacterial polyhydroxyalkanoate, $A d v$. Drug Delivery Rev., 2001, 53(1), 5-21.
7 G. Q. Chen, A microbial polyhydroxyalkanoates (PHA) based bio- and materials industry, Chem. Soc. Rev., 2009, 38, 24342446.

8 D. B. Hazer, E. Kılıçay and B. Hazer, Poly(3hydroxyalkanoate)s: Diversification and biomedical applications: A state of the art review, Mater. Sci. Eng., C, 2012, 32(4), 637-647.

9 E. Bugnicourt, Polyhydroxyalkanoate (PHA): Review of synthesis, characteristics, processing and potential applications in packaging, eXPRESS Polym. Lett., 2014, 8(11), 791-808.

10 A. Hoffmann, S. Kreuzberger and G. Hinrichsen, Influence of thermal degradation on tensile strength and Young's modulus of poly(hydroxybutyrate), Polym. Bull., 1994, 33(3), 355-359.

11 N. Grassie and E. J. Murray, The thermal degradation of poly $(-(d)-\beta$-hydroxybutyric acid): Part 1 -Identification and quantitative analysis of products, Polym. Degrad. Stab., 1984, 6(1), 47-61.

12 D. Kai and X. J. Loh, Polyhydroxyalkanoates: Chemical Modifications Toward Biomedical Applications, ACS Sustainable Chem. Eng., 2014, 2(2), 106-119.

13 R. Nigmatullin, P. Thomas, B. Lukasiewicz, H. Puthussery and I. Roy, Polyhydroxyalkanoates, a family of natural polymers, and their applications in drug delivery, J. Chem. Technol. Biotechnol., 2015, 90(7), 1209-1221.

14 G. R. Saad, Y. J. Lee and H. Seliger, Synthesis and characterization of biodegradable poly(ester-urethanes) based on bacterial poly(R-3-hydroxybutyrate), J. Appl. Polym. Sci., 2002, 83(4), 703-718.

15 Z. Špitalský, I. Lacík, E. Lathová, I. Janigová and I. Chodák, Controlled degradation of polyhydroxybutyrate via alcoholysis with ethylene glycol or glycerol, Polym. Degrad. Stab., 2006, 91(4), 856-861.

16 B. Hazer, Amphiphilic Poly(3-hydroxy alkanoate)s: Potential Candidates for Medical Applications, Int. J. Polym. Sci., 2010, 2010, 1-8.

17 D. Kai and X. J. Loh, Polyhydroxyalkanoates: Chemical Modifications Toward Biomedical Applications, ACS Sustainable Chem. Eng., 2014, 2(2), 106-119.

18 D. Xue, W. Lv and H. Zhang, Telechelic diols from polyhydroxybutyrate via alcoholysis with ethylene glycol or glycerol, Proceedings of the 2015 2nd International Conference on Machinery, Materials Engineering, Chemical Engineering and Biotechnology (MMECEB 2015), Atlantis Press, 2016.

19 C. Lauzier, J.-F. Revol, E.-M. Debzi and R. H. Marchessault, Hydrolytic degradation of isolated poly( $\beta$-hydroxybutyrate) granules, Polymer, 1994, 35(19), 4156-4162.

20 G. Adamus, W. Sikorska and M. Kowalczuk, Sequence Distribution and Fragmentation Studies of Bacterial Copolyester Macromolecules: Characterization of PHBV Macroinitiator by Electrospray Ion-Trap Multistage Mass Spectrometry, Macromolecules, 2000, 33(16), 5797-5802.

21 E. Žagar, A. Kržan, G. Adamus and M. Kowalczuk, Sequence Distribution in Microbial Poly(3-hydroxybutyrate-co-3- 
hydroxyvalerate) Co-polyesters Determined by NMR and MS, Biomacromolecules, 2006, 7(7), 2210-2216.

22 S. Nguyen, G. Yu and R. H. Marchessault, Thermal Degradation of Poly(3-hydroxyalkanoates): Preparation of Well-Defined Oligomers, Biomacromolecules, 2002, 3(1), 219-224.

23 M. Kawalec, G. Adamus, P. Kurcok, M. Kowalczuk, I. Foltran, M. L. Focarete and M. Scandola, Carboxylate-Induced Degradation of Poly(3-hydroxybutyrate)s, Biomacromolecules, 2007, 8(4), 1053-1058.

24 J. M. Bergamaschi, E. J. Pilau, F. C. Gozzo and M. I. Felisberti, Synthesis of Polyurethane from Poly(3hydroxybutyrate) and Poly(p-dioxanone): Molar Mass Reduction via Sodium Borohydrate, Macromol. Symp., 2011, 299-300(1), 10-19.

25 M. Kwiecień, G. Adamus and M. Kowalczuk, Selective Reduction of PHA Biopolyesters and Their Synthetic Analogues to Corresponding PHA Oligodiols Proved by Structural Studies, Biomacromolecules, 2013, 14(4), 1181-1188.

26 S. Montoro, Master Dissertation, Universidade de São Paulo, Lorena, 2005.

27 H. C. Brown, H. l. Schlesinger and A. B. Burg, Hydrides of Boron. XI. The Reaction of Diborane with Organic Compounds Containing a Carbonyl Group, J. Am. Chem. Soc., 1939, 61(3), 673-680.

28 R. Vasita, K. Shanmugam and D. S. Katti, Improved Biomaterials for Tissue Engineering Applications: Surface
Modification of Polymers, Curr. Top. Med. Chem., 2008, 8(4), 341-353.

29 K. Anselme, L. Ploux and A. Ponche, Cell/Material Interfaces: Influence of Surface Chemistry and Surface Topography on Cell Adhesion, J. Adhes. Sci. Technol., 2010, 24(5), 831-852.

30 I. Kwiecień, I. Radecka, M. Kwiecień and G. Adamus, Synthesis and Structural Characterization of Bioactive PHA and $\gamma$-PGA Oligomers for Potential Applications as a Delivery System, Materials, 2016, 9(5), 307.

31 M. Kwiecień, I. Kwiecień, I. Radecka, V. Kannappan, M. R. Morris and G. Adamus, Biocompatible terpolyesters containing polyhydroxyalkanoate and sebacic acid structural segments - synthesis and characterization, RSC Adv., 2017, 7(33), 20469-20479.

32 G. Adamus, Molecular Level Structure of $(R, S)-3$ Hydroxybutyrate/( $R, S)$-3-Hydroxy-4-ethoxybutyrate Copolyesters with Dissimilar Architecture, Macromolecules, 2009, 42, 4547.

33 Y. Arima and H. Iwata, Effects of surface functional groups on protein adsorption and subsequent cell adhesion using self-assembled monolayers, J. Mater. Chem., 2007, 17, 4079-4087.

34 A. Zühlke, B. Röder, H. Widdecke and J. Klein, Synthesis and application of new microcarriers for animal cell culture. Part I: Design of polystyrene based microcarriers, J. Biomater. Sci., Polym. Ed., 1993, 5(1-2), 65-78.

35 P. Viccellio, Emergency Toxicology, Lippincott-Raven Publishers, 1998, p. 293. 